\title{
Achtjähriges Verweilen eines Schrotkornes in der vorderen Augenkammer.
}

\author{
Oasnistische Mittheilung. \\ Ton \\ Dr. Burstenbinder, \\ Augenarzt in Hamburg.
}

Mit 泾Af. XVI.

Die Resultate, welche Leber(1) mit seinen Versuchen über die Wirkung der Fremdkörper im Auge erhalten hat, und die Erfahrung, dass selbst ein chemisch völlig indifferenter, aseptisch in das Auge gelangter Körper, auch wem er jahrelang unschädlich bleibt, doch bei irgend einer Veranlassung die Ursache von Reizzuständen und neuen Entzündungen des verletzten, sowie sympathischer Reizung des anderen Auges abgeben, und so zu stärkerer Beeinträchtigung der Functionen führen kann, machen es uns zur Bedingung, in jedem Falle, in dem sich ein Fremakörper nach einer Verletzung im Auge befindet, denselben baldigst $z \mathrm{u}$ entfernen, und so das Auge ror weiteren schädigenden Momenten nach Möglichkeit zu bewahren. Andererseits wird in vielen Fällen durch die Verletzung selbst oder eine hinzugetretene Infection die Erblindung oder gar Zerstörung des Bulbus herbeigeführt. Wir diurfen uns daher nicht wundern, in der Literatur nur verhältnissmässig wenige Beobachtungen niedergelegt zu finden, in denen bei noch sehtiichtigen Augen ein Fremdlö̈rper jabrelang im Bulbusinnern verweilte, ohne schwerere Störungen zu verursachen und das Sehvermögen erheblich zu schädigen.

Am gïnstigsten liegen die Verhältnisse in den Fällen, wo der Fremakörper in die Linse gelangt, und durch schnellen Schluss der Kapselwunde das Entstehen einer Totalcataract verhindert wird. Es sei nur an den von Wagenmann(2) mitgetheilten Fall erinnert, wo ein Kupferstiuck 25 Jahre in der Linse sass, ohne eine Störung zu bewirken. 
Nicht ganz so günstig gestalten sich die Bedingungen bei Lage des Fremdkörpers in der vorderen Kamimer. Alle in der Literatur bis 1890 niedergelegten Beobachtungen von Fremdkörpern in der Vorderkammer und ihrer Umgebung sind in den Arbeiten von Landmann(3), Blessig(4) und Franke(5) zusammengestellt. Hier sollen nur die wenigen Mittheilungen kurz aufgetührt werden, in denen der Fremdkörper längere Zeit in der vorderen Kammer lag, obne besondere schädliche Wirkungen für das. noch sehtiichtige Auge zu entfalten.

Betreffs der Cilien, welche ziemlich häufig in der Vorderkammer beobachtet worden sind, und, obne die mindeste Belästigung zu verursachen, bis zu zehn Jahren dort verweilt haben [Pagenstecher(6), Peschel(7)], verweise ich auf die Arbeit yon Müller(8), da die Cilien eine etwas gesonderte Stellung unter den Fremdkörpern einnehmen.

Richardson (9). Einem Soldaten wurden 1813 durch Zersplitterung eines Baumes durch eine Kanonenkugel in das eine Auge drei Holzsplitter eines Astes eingetrieben, während das andere Auge total zerstört wară. Damals bestand eine vorübergehende Entzündung. Seit 46 Jahren ist das Auge reizlos. Die Iris ist auf einen schmalen Streifen reducirt. Die Splitter sind als drei weissliche Körperchen, von zarter Haut umkleidet und mit einigen Pigmentkörnehen bestreut, in der Vorderkammer sichtbar. Sehvermögen gut.

Jacob (10) berichtet über einen Fall, in dem ein sehr scharfer, 1/4 Zoll langer und $3 / 6$ Zoll dicker Steinsplitter in der Vorderkammer erst nach vier Jahren erheblichen Reizzustand veranlasste.

Saemisch (11). Ein Mann erhielt vor zwölf Jahren eine Verletzung beim Steinklopfen, damals leichte Entzündung mit Herahsetzung des Sehvermögens bis auf Lichtschein. In den zwölf Jabren keinerlei krankhafte Erscheinungen, bis vor drei Wochen eine schmerzhafte Entzündung einsetzte. In der Vorderkammer ein scharfkantiger selwarzer Körper, von einer leichten grauen Hülle umgeben, der durch eine strangförmige Verbindung mit der Cataracta secundaria in der Kammer schwebend erhalten wird. Extraction des Fremdkörpers, eines Basaltstückes von $5 \mathrm{~mm}$ Länge und $2^{1 / 2} \mathrm{~mm}$ Dicke. Die Hülle besteht zum grössten Theil aus Linsenkapsel. Visus des aplakischen, mit Cataracta secundaria behafteten Auges wenige Tage nach der Operation bestelt in Fingerzählen in einigen Fuss.

In Friedinger's (12) Fall verweilte ein Steinsplitter bereits 19 Jahre in der vorderen Kammer. Jalıre hindureh waren oft reeidivirende Entzündungen, stets mit heftigen Kopfselımerzen combinirt, aufgetreten. Zur Zeit bestand wieder Iritis mit Ciliarneurose und starker Herabsetzung des Sehvermögens. Beim Versuch, den Splitter zu extrahiren, musste man sich mit der Iridektomie begnügen. Nach derselben war der Splitter in der Vorderkammer gut sichtbar, und das Sehvermögen ward fast wieder normal.

Von längerem Verweilen von Glassplittern in der vorderen Augenkammer liegen fünf Beobachtungen vor, doch sollen die zwei von Bickerton (13 und 14) mitgetheilten Fälle dieser Aufzählung nicht eingereiht werden, da es fraglich bleibt, ob sie allen hier gestellten Bedingungen 
entsprechen. Die Originalarbeiten waren mir leider nicht zugänglich, und so muss iel mich mit einem knrzen Referate begnügen, das nur die zehn- resp. seehsjährige Daner des Aufenthaltes der Glassplitter in der Vorderkammer anführte.

Höring(15). Ein Glassplitter latte über fün Jahre in der Vorderkammer gelegen, schmerzhafte Entzündungsanfälle und pannöse Trübnngen der Hornhaut hervorgerufen, his es Jäger gelang, ihn zu entfernen. Seltschäre kaum geschwächt.

Critchett (16). Glassplitter, frei beweglich, seit 16 Jahren in der Vorderkammer. Häufig leichte Entzindung. Bei einem Extractionsversuch war der Splitter nach Alufuss des Kammerwassers nicht mehr zu finden, er war offenbar mit dem Kammerwasser entleert. Nach einem Jahr war das Auge normal.

Wagenmann (17) berichtet, dass ein $4 \mathrm{~mm}$ langer, $2 \mathrm{~mm}$ breiter und $1 \mathrm{~mm}$ dicker Glassplitter die ersten Monate gut im Auge vertragen wurle, damn traten Anfälle yon Röthung mit Lichtseheu ein und eine stetic zunehmende parenchymatöse Hornhauthribung, gefolgt von Abnahme des Sehvermögens. Nach der, ein Jahr nach der Verletzung vorgenommenen Extraction gingen die Veränderungen zurüek, und das Sehvermögen stellte sich znm grössten Theil wieder leer.

Enapp (18) entfernte mittels Haken ein Zündhütchenstïck, das ror einem Jahre in das Auge gelangt war und damals vorübergehend Entzindnngserscheinungen hervorgerufen hatte. Seit zwei Wochen bestand recidivirende Iridocyclitis, Das Kupterstück lag als längliches, graubrannes Innötchen am Boden der Vorderkamner. Nach der Extraction trat Rück. bildung der Entzündungserscheinungen und Heilung mit relatir gutem Visus ein. zureihen.

Von Eisen- und Stallsplittern wären zwei Beobachtungen hier an-

Landesberg (19) ward zwecks Brillenbestimmung von einem Patienten consultirt, bei dem seit zehn Jahren ein Eisensplitter in Humor aqueus Hottirte. Wiederholt waren lejelite Entzündungszuntände des verletzten Auges aufgetreten, die aber nur wenige Tage anhielten, geringe Beschwerden rerursachten und spontan zurüekgingen. Auf der Membrana Descemeti zwei kleine Häutchen von punktförmigen Besehlägen; Iris solmutzig gran, Zeiehnung verwaschen. Humor aqueus klax. Ein kleiner schwarzer, spitz zulaufender Körper gehwimmt frei im Karnmerwasser und folgt allen Bewegungen des Auges. Zwei fadenförmige lintere Synechien. Einzelne äquatoriale Chorioidealatrophien. Sehvermögen ${ }^{15 / 20}$. Durch Paracentese ward en " $/ 2$ " langer, keilförmiger, rostiger Eisensplitter entfernt.

Topolansky (20). Ein ungefälı $3 \mathrm{~mm}$ langes Strïck Eisen, nieht eingekapselt, bewegte sich seit 22 Jaluren frei in der vorderen Kammer herum. Es bestanden keine Entzlindungserscheinungen. Beim Abwärtmeigen des Loopfes rolt der Fremdkörper aus dem unteren Kanmerfalz hervor, kollert beim Aufrichten uther die Descemet'sche Membran wieder linunter" und verselwindet im Kammerfalz. Inis etwas verwaschen, zeigt einen Stich ins Olivgr'ine. Kammerwasser klar. Der Deseemet'sehen Membran sitzen triibe Fleckehen von gelblicher Färbung auf, mit unregelmässiger Begrenzung.

Die nächsten Mittheilungen betrefien Schrotkörner. 
Salomon (21) berichtet von einem Schrotkorn, das, dureh plastisches Exsudat abgekapselt, fünf Jahre in der Vorderkammer lag, ohne Schmerzen oder Besehwerden zu machen oder das Sehvermögen zu schädigen.

Graefe (22) erzählt, dass einem Fischer ein Schrotkorn ins Auge drang, dort eine Entzündung des Auges und die Resorption der Linse hervorgerufen habe, darauf sei der Fremdkörper in die vordere Kammer gefallen, wo er, von Flocken umgeben, sechs Jahre liegen blieb, olne den Bulbus in seiner Integrität and Function zu störea.

Der letzte Fall wird von Kipp (23) mitgetheilt, doch konnte ich nur die Angabe finden, dass ein Schrotkorn acht Jahre in der Vorderkammer sass.

Ich bin in der Lage, diesen wenigen Beobachtungen einen weiteren Fall anfuigen zu können, welchen ich letzthin durch Zufall zu untersuchen Gelegenheit hatte.

Anamnese: Sch., Quaiarbeiter, früher nie wesentlich augenkrank, elhielt im Sommer 1893, im Alter von 16 Jahren, einen Schrotschuss in die Gegend seines linken Auges. Der Schuss war aus einer Enfernung von ca. finf Schritt etwas von rechts her abgefeuert. Nähere Mittheilungen uber den damaligen Befund und die Krankenhausbehandlung stehen mir leider nicht zu Gebote, so dass idh nur die kurzen Angaben des Patienten anführen kann. Er giebt an, vor der Verletzung auf beiden Augen gleich gut gesehen und nicht geschielt zu haben. Das linke Auge sei von einem Schrotkorm durchbohrt worden. Mehrere Schrote aus der Haut wurden en̉tfernt. Als die Bulbuswunde verheilt war, hat er bemerkt, dass er schlechter sah, schielte und doppelt sah. Wegen der Doppelbilder war er die erste Zeit genöthigt, eine Klappe vor dem schielenden Auge zu tragen, loch leinte er bald das Bild des sehschwächeren Auges unterdrüicken, und ging seitdem seiner Arbeit ohne Klappe ungestört nach. Niemals liat er in den verflossenen $7^{3 / 4}$ Jahren irgend eine Störung oder Entzündung des Auges bemerkt. Die Doppelbilder kann er jeder Zeit sich sehr leicht zum Bewusstsein bringen.

Status praesens: 15 . III. 1901.

Rechtes Ange äusserlich und ophthalmoskopisch normal. E. S. $=6 / 6$. Kleinste Schrift gelesen. Gesichtsfeld normal. In den vordersten Linsenschichten feine punkt- und fleckförmige Trübungen, die bei Lupenbeleuchtang einen schönen, intensiv grünlich-blanen Reflex geben Cataracta caerulea congenita.

Links besteht Einwärtsschielen mittleren Grades. Der Bulbus ist nach allen Seiten frei beweglich, eine Muskellähmung ist nicht nachweisbar.

Patient giebt glejchnamige Doppelbilder an, deren Distanz sich bei allen Augenstellungen gleich bleibt. Es handelt sich also um Strabismus conver'gens concomitans. Die Lidspalte ist ein wenig verengt durch geringes Herabhängen des Oberlides. Das Auge selbst ist absolut reizlos. Die Hornhaut weist nasal am Limbus in der Höle des horizontalen Meridians eine ungefälr erbsengrosse leukomatöse Tribung auf, an der ein ziemlich peripher gelegener, feiner Iriszipfel adhärent ist. Das Kammerwasser ist vollkommen klar. An der tiefsten Stelle ragt aus der Kammerbucht ein dunkler Körper 
hervor; der auch bei Lageänderungen des Kopfes in seiner Stellung behart. Bei Lupenbetrachtung erkennt man, dass offenbar ein kugeliger Körper hier eingebettet ist, man sieht ungefähr die obere Hälfte einer kleinen Kugel von brannschwarzer Farbe und glatter Oberfläehe, die den Ciliartheil der Iris nach hinten ausbuehtet, and anf der einige feine grawweissliche Gewebsflocken lagern. Die zurïckgewölbte Partie der Regenbogenhaut ist ganz leicht atrophisch. Von Gewebsauflagerung in der Kammerbucht oder auf' der Iris ist nichts zu entdecken. Die Pupille ist nicht ganz rund, leicht anfrecht oval, frei beweglich. Keine bestehenden noch Reste früherer Synechien. Reaction der Pupille prompt. Die Linse weist nasal, entsprechend der leukomatösen Hornhanttrübung, eine cireumserjpte saturitere Trübung auf, an die anschliessend feine punktförmige Trúbungen nach der Mitte und nach unten zu bis an die Grenze des Pupillargebietes sich ausbreiten. Diese feinen Trübungen scheinen zum grössten Theil der vorderen, zum kleinen Theil aber auch der hinteren Corticalis anzugehören. Dieht hinter der Linse, etwas unterhalb der Horizontalen, flotirt im vordersten Glaskörperabschnitt eine längliche grane Glaskörperflocke.

Der Angenhintergrund weist die Verändernngen einer Retina-Chorioidealruptur anf. Am unteren Ende der Papille (a. B.) beginnend, erstreckt sich in einer Länge von ca. fünf Papillendurchmessern und grössten Breite von zwei Papillendiametern die Ruptur leicht bogenformig nach innen unten als leuchtend weisser Fleck, an den Rändern zum Theil mit dickem, klumpigem Pigment umgeben. Retinalgefässe ziehen nur an der äussersten Stelle innen unten uber die Ruptur hinweg. An den Rundern sieht man stellenweise feine, grane, convergüende Streifen, offenbar die nmgestülpten zusammengelegten Ränder der eingerissenen Netzhaut. Im iubrigen Angenhintergyund keine sichtbaren Ferïnderungen.

Das Sehvermögen des Auges betrügt mit - 1,3̆ Di. S. $=6 / 18^{6}$ Das Gesichtsfeld weist einen Defect fast der ganzen oberen Gesichtsfeldhälfte anf.

Bei länger dauernder Cntersuehung tritt leichte ciliare Injection am unteren Hornhautrand auf, mit Thränen des Auges, Erseheinungen, die sehnell wieder sehwinden.

Jeder $Z$ weifel über die Satur des im Kammerfalz eingebetteten Frendkörpers ward durch eine Röntgenaufinahme gehoben, die deutlich einen runden Sehatten von der Grösse eines Sehrotkornes in der Gegend der Vorderkammer erkennen lässt $(\mathrm{s}, \mathrm{Fig})$. ( $\mathrm{Zu}$ genaueren Orientirmg ist vor der Aufnahme dem Orbitalbogen ein Bleidraht angebogen nnd fixirt worden, der sich auf dem Bilde deutlich als dunkler Streifen abhebt.) Das Bild zeigt ferner, dass atusser diesern, im Auge gelegenen, noch viele Schrotkörner in der Stirn-, Nasen- und Wangengegend, und am Orbitalbogen sitzen mûssen, die sich denn anch zum Theil sehr gut palpiren liessen, sowie, dass zwei Schrote in der Tiefe, offenbar an der inneren Orbitalwand, gelegen sind. (Die Sohatten dieser beiden letzten sind von beträchtlich gevingerer Schärfe.)

Epikrise. Es handelt sich in diesem Falle um ein Auge, das, vor nahezu acht Jahren von einem Schrotkorn durchbohrt, seit dieser Zeit den Fremakörper in der Eammerbucht beherbergt, keine Zeichen 
früherer oder gegenwärtiger Entzündung aufweist, absolut reizlos ist und eine Herabsetzung des Sehvermögens nur insoweit erkennen lässt, als den übrigen durch die Verletzung gesetzten Veränderungen entspricht.

Was das in das Auge gelangte Projectil betrifft, so reichte seine Kraft gerade nur zur Perforation der Hornhaut aus, nachher muss es bald in die Kammerbucht hinabgeglitten sein. Ob die Regenbogenhaut mit perforirt worden ist, bleibt unentschieden. Dagegen spricht die Grösse der Linsentrübung entschieden gegen ein zeitweiliges Eindringen des Schrotkornes in die Linse selbst, es wird vielmehr durch den Anprall ein kleiner Einriss der Kapsel stattgefunden haben, der sich schnell wieder schloss, so dass die Trübung der Linse circumscript und von geringer Ausdehnung blieb.

Die Aderhaut-Netzhautruptur muss durch Contrecoup entstanden gedacht werden.

Einer Erklärung bedarf noch die Entstehung des Strabismus concomitans. Ich möchte annehmen, dass die oben erwähnten, in der Nähe der inneren Orbitalwand gelegenen Schrotkörner die Ursache des Strabismus sind, indem sie eine Verletzung der Sehne oder des Muskelbauches des Rectus internus beim Eindringen in die Orbita bewirkten, und dass dann durch die narbige Schrumpfung eine Verkiurzung des Muskels und so der Strabismus entstand. In ähnlicher Weise liesse sich die leichte Ptosis durch eine geringe Läsion des Levator oder eines Nervenastes erklären, herbeigetührt durch eins der am oberen Orbitalbogen gelegenen Schrote.

Das Fehlen von Veränderungen, die auf eine frühere oder jetzige Entzïndung hätten zurückgeführt werden können, beweist einerseits, dass das Schrotkorn steril war, als es den Bulbus perforirte, andererseits, dass Blei sich in der vorderen Kammer ziemlich indifferent verhält.

Auf die sterilisirende Kraft der Explosion bei Schrotschüssen haben besonders Tornatola (24), Lodato(25) und Ovio (26) hingewiesen. Ovio erwies die sterilisirende Kraft der beim Schuss entstehenden Hitze direct durch Experimente. Er inficirte Schrotkörner mit Reinculturen von Staphylococcus pyogenes und schoss sie gegen Kaninchen und Nährgelatine ab. Die Wunden der Kaninchen heilten reactionslos, und die Gelatine blieb steril.

Dieselben Versuche mit Milzbrandsporen gaben allerdings ein ungünstigeres Resultat. Die Kaninchen gingen an Milzbrand zu Grunde, und in der Gelatine entwickelten sich Culturen. Da die 
Milzbrandsporen zu den am schwersten abzutödtenden pyogenen Keimen gehören, die wir kennen, die Reinculturen von Staphylococcus pyogenes aber total durch die Hitze beim Schuss abgetödtet werden, darf man annehmen, dass in den meisten Fällen ein durch Schuss in das Auge gelangtes Schrotkorn aseptisch ist.

Was den zweiten Punkt anlangt, möchte ich den darauf bezüglichen Passus aus dem bekannten Werk von Leber (1) citiren:

„Fremdkörper aus Blei, verhalten sich in der vorderen Kammer des Kaninchens ziemlich indifferent, doeh weniger als gleich grosse Fremdkörper ans Gold oder Glas, indem es in ihrer Umgebung zu, schon während des Lebens erkennbaren, geringen Gewebsveränderungen entzündlicher Natur kommt, welche aber im weiteren Verlauf wieder rüekgängig werden."

"Die geringe Wirkung erklärt sich ohne Zweifel dadurch, dass das Blei, welches in löslicher Form stark giftig auf die Elemente des Körpers wirkt, hier nicht in merklicher Menge zur Lösung kommt. Bei der Unlöslichkeit seines Carbonates in kohlensäurelialtigen Flüssigkeiten felılen die Bedingungen, um eine noch so verdünnte Bleilösung in genügender Menge den benachbarten Geweben und Blutgetässen zuzufuhren; der Lremdkörper würde, auch wenn đie Kohlensüure des Kammerwassers anfangs etwas auf ibn einwirkte, sich bald mit einer dunnen Schicht von kohlensaurem Bleioxyd bedecken, welche die weitere Einwirkung der Kohlensäure verhinderte. Dieser Einfluss kann übrigens nur ein sehr geringer sein, weil die eingeführten Bleistücke thr metallisches Aussehen, wenn auch mit einer gewissen Abnahme des Glanzes, behielten."

Sowohl die an Kaninchen angestellten Versuche wie die Beobachtungen am Menschen beweisen also, dass Blei sich in der vorderen Kammer nahezu indifferent verhält, und aus der oben aufgeführten Zusammenstellung ist zugleich zu ersehen, dass es bei Weitem unschädlicher ist als Glas. Alle drei Fälle von Glassplitterverletzung führten zu Entziłhnlungen, und zwei zu progressiver parenchymatöser Cornealtrïbung, wäbrend in den' bekannt gewordenen Fällen, in denen Schrotkörner in der Vorderkammer verweilten, auch nach vielen Jahren keine Complicationen bekannt geworden sind.

\section{Literaturverzeichniss.}

1) Leber, Die Entstehung der Entzhndung. Leipzig 1891. Wilhelm Engelmann.

2) Wagenmann, v, Graefe's Arch. f. Ophthalm. XLIV. Bd. 2. S. 272.

3) Laudmann, v. Graefe's Arch. 1. Ophthalm. XXVIL. Bd. 2.

4) Blessig, Klin. Monatsbl. f. Augenheilk. 1890. S. 179.

5) Franke, v. Graete's Arch. f. Ophthalm. XXX. Bd. 1.

6) Pagenstecher, citirt r. Graefe. r. Graefe's Arch.f. Ophthalm. TH. Bd. 2. S. 139. 
Achtjähriges Verweilen eines Schrotkornes in der vorderen Augenkammer. 483

7) Peschel, Centralblatt für praktische Angenheillkmde. 1887. S. 113.

8) Müller, Wiener klin. Wochenschr. 1894. S. 232.

9) Richardson, Dublin. quarterly Joum. 1859. XXVIII. p. 320.

10) Jacob, Dublin. med. Presse. 1846. December.

11) Saemisch, Krlin. Monatsbl. f. Angenheilk. 1865. S. 46.

12) Friedinger, Wiener med. Wochenschr. 1878. XXVIII. S. 352.

13) Bickerton, Brit. med. Joum. 1888. April. p. 896.

14) Bickerton, Brit. med. Jomru. 1888. June. p. 1215.

15) Höring, Ammon's Zeitschrift f. Ophthalm. 1833. III. S. 103.

16) Critchett, Ophthalm. Hosp. Reports. 1859. I. p. 264.

17) Wagenmann, v. Graefe's Arch. f. Ophthalm. XL. Bd. ๖. S. 180.

18) Knapp, Arch. f. Augenheilk. 1880. IX. Bd. 2.

19) Landesberg, Klin. Monatsbl. f. Augenheilk. 188\%. S. 320.

20) Topolansky, Wiener med. Wochenschr. 1895. XLV. S. 1894.

21) Salomon, v. Ammon's Zeitschrift f. Ophthalm. 1830. I. S. 336.

22) Graefe, Graefe-Walther's Journ. XIV. S. 157.

23) Kipp, Americ. Journ. of Oplithalm. 1884. I. p. 103.

24) Tornatola. Arch. di Ottalm. 1896. III. p. 350 .

25) Lodato, Arch. di Ottalm. 1895. II. Fase. 9-10.

26) Ovio, Revue générale d'Ophthalm. 1895. p. 305. 


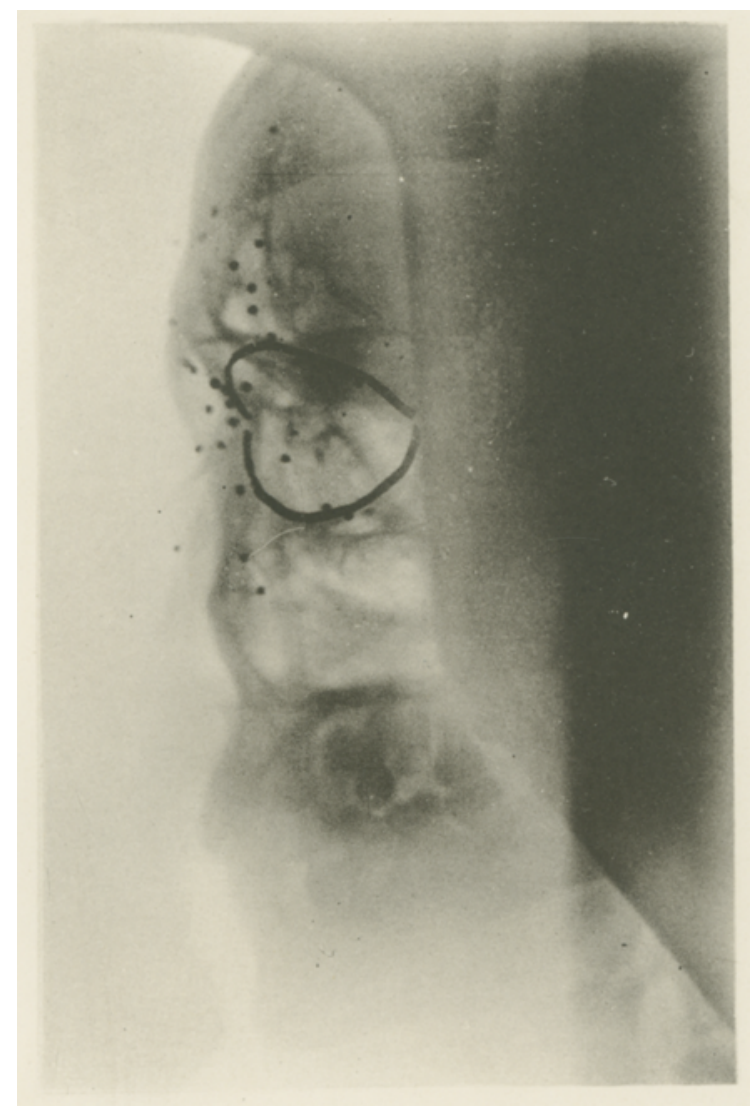

Verlag v. Wilhelm Engelmann in Leipzig. 